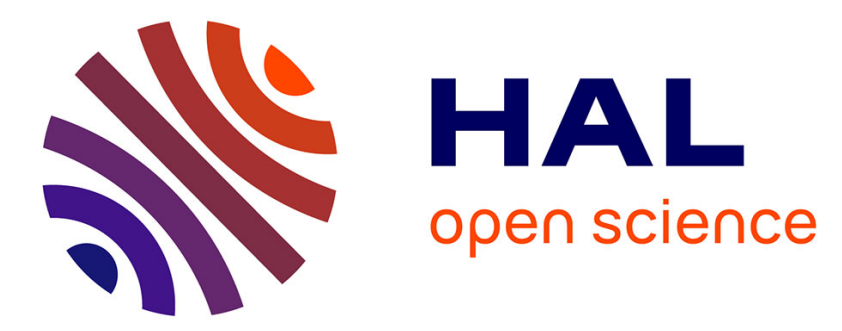

\title{
Le rêve de la fleur de prunier : la transmission généalogique chez Dōgen (1200-1253) en Chine
}

Frédéric Girard

\section{To cite this version:}

Frédéric Girard. Le rêve de la fleur de prunier : la transmission généalogique chez Dōgen (1200-1253) en Chine. Comptes-rendus des séances de l'Académie des inscriptions et belles-lettres, 2009, 153 (3), pp.997-1017. 10.3406/crai.2009.92571 . hal-02537103

\section{HAL Id: hal-02537103 https://hal.science/hal-02537103}

Submitted on 8 Apr 2020

HAL is a multi-disciplinary open access archive for the deposit and dissemination of scientific research documents, whether they are published or not. The documents may come from teaching and research institutions in France or abroad, or from public or private research centers.
L'archive ouverte pluridisciplinaire HAL, est destinée au dépôt et à la diffusion de documents scientifiques de niveau recherche, publiés ou non, émanant des établissements d'enseignement et de recherche français ou étrangers, des laboratoires publics ou privés. 


\section{Le rêve de la fleur de prunier : la transmission généalogique} chez Dōgen (1200-1253) en Chine (note d'information)

Frédéric Girard

\section{Citer ce document / Cite this document :}

Girard Frédéric. Le rêve de la fleur de prunier : la transmission généalogique chez Dōgen (1200-1253) en Chine (note d'information). In: Comptes rendus des séances de l'Académie des Inscriptions et Belles-Lettres, 153e année, N. 3, 2009. pp. 997-1017;

doi : https://doi.org/10.3406/crai.2009.92571

https://www.persee.fr/doc/crai_0065-0536_2009_num_153_3_92571

Fichier pdf généré le 19/07/2018 


\title{
NOTE D'INFORMATION
}

\author{
LE RÊVE DE LA FLEUR DE PRUNIER : LA TRANSMISSION \\ GÉNÉALOGIQUE CHEZ DŌGEN (1200-1253) EN CHINE, \\ PAR M. FRÉDÉRIC GIRARD
}

Les Japonais sont, on le sait, d'incorrigibles rêveurs et la documentation sur le sujet est telle qu'elle défie toute classification qui se voudrait exhaustive. Les études qui s'y sont essayé sont pourtant légion, qui laissent transparaître des angles d'approche multiples. Nous nous arrêterons ici à un cas d'espèce, celui du moine 道元 Dōgen (1200-1253), qui a vécu au début du Moyen Âge.

Des rêves, au Japon, comme partout ailleurs, on ne parle point : dangereux ou objets de tabou, ils sont relégués dans l'intimité de la psychè, enfouis dans une tradition orale ou secrète, consignés dans des carnets à usage interne et inaccessibles au public, parfois notés sur des revers de manuscrits, en toute occurrence livrés aux seuls initiés. Ils influent sur le cours des événements et des choses : ils sont des principes actifs, logés dans le for intérieur du sujet qui en est le seul dépositaire, faute de quoi ils perdent leur efficacité. Une fois dévoilés, ils ne sont plus que matière et idée inertes.

Cette redoutable efficacité des rêves est, sur un plan religieux, celui qui nous intéresse ici, le prélude ou la résultante d'une discipline inflexible, d'une concentration de l'esprit à toute épreuve, qui a pour pendant des modes d'expression faits de non-dits, par lesquels les Japonais s'illustrent depuis les temps les plus reculés.

Pour notre propos, afin de mieux circonscrire le rêve de Dōgen que nous allons décrire - à vrai dire l'unique qu'on puisse lui attribuer avec certitude -, nous ferons état des types de rêves et de relations oniriques chez les religieux de son époque, à la lisère de la fin de l'Antiquité et du début du Moyen Âge. Les temps sont marqués par des guerres civiles incessantes et à rebondissements mettant sur le devant de la scène nobles et guerriers, et l'on a noté que, statistiquement, les relations oniriques sont, au Japon, tous âges confondus, particulièrement fécondes en ceux de troubles militaires.

Dans ses orientations, l'histoire du bouddhisme japonais est, peut-on admettre, marquée par un contraste entre deux traditions : 
l'une, « exotérique », dans laquelle l'exposé positif des enseignements va de pair avec une ascèse qui tend à nier les rêves, expression de la soif inextinguible et répréhensible de l'homme; et l'autre, « ésotérique », qui, à l'inverse, révèle les intentions cachées de ces enseignements, souvent déposées dans des traditions orales, et revalorise les désirs passionnels comme principes moteurs sublimant les activités humaines, parmi lesquels les rêves occupent une place de choix. Ces deux aspects opposés peuvent bien entendu se conjuguer. À la première tendance, ressortit le Zen auquel appartient Dōgen 道元, qui semblait peu enclin à rêver, et à la seconde tendance se rattache son quasi-contemporain Myōe 明恵 (1173-1232), qui a tenu sa vie durant une chronique journalière de ses rêves, qui constituait un bien à la fois personnel et collectif.

L'étymologie courante du caractère désignant le rêve, meng 夢, associe les visions oniriques à une maladie, celle d'un patient qui est assailli par de mauvais songes, tout en étant couché ou appuyé tant bien que mal sur son lit $^{1}$. En outre, la tradition religieuse aussi bien du taoïsme que du bouddhisme assure que le sage n'a par définition pas de rêve : il est exempt de déficience mentale et le courant de son psychisme ne connaît ni aspérité ni discontinuité entre veille et sommeil.

Chez Myōe en particulier, on voit se développer une analyse des visions oniriques, de plusieurs points de vue concomitants et confluents, en accord d'ailleurs avec les conceptions de son époque :

1. Les rêves reflètent des impressions diurnes, des expériences de la vie vigile, réorganisées dans des récits oniriques. Ils sont expliqués comme s'intégrant à une continuité causale, allant de la cause à des effets, homogène et sur le même plan que les phénomènes de la vie psychique éveillée. « Les actes cognitifs accomplis en rêves sont exactement comme des actes accomplis dans la vision et l'audition (dans les processus cognitifs) », nous confie le jeune Myōe, avant de se mettre à tenir son Journal de rêves ${ }^{2}$.

2. Les rêves qui, par nature, ne sont pas différents des phénomènes psychiques diurnes, présentent des signes, sō 相, qui sont

1. Voir à ce sujet les travaux de J.-P. Diény, résumés dans l'Annuaire de l'École Pratique des Hautes Études, IV ection, année 1996-1997. Liou Wenying 劉文英 (Chūgoku no yume handan 中国の夢判断 [Oniromancie chinoise], 1997) dénie cette étymologie et est d'opinion que les graphies archaïques désignent simplement le fait de voir quelque chose qui ne soit pas clair, notamment durant le sommeil.

2. Le passage du Texte de vœu d'intention particulière, Zuii betsu ganmon 隨意別願文 cité ici date du début de sa chronique onirique, lorsque Myōe avait 18 ans. 
l'envers de représentations, $s \bar{o}$ 想, et se résolvent dans la conception que toute chose n'est que représentation mentale, ou que du mental, yuishin 唯心. Les graphies des caractères $s \bar{o}$ désignant les signes cognitifs et la représentation de ces signes ne se différencient que par l'absence ou la présence du caractère désignant le mental ou l'esprit : les deux processus sont conçus comme n'étant que les deux versants d'un même phénomène. Ce tout s'applique aussi bien au monde phénoménal des illusions, qu'à celui supra-mondain de l'Éveil et du Nirvāṇa, si bien que les rêves sont des signes tant de la causalité mondaine - ils s'inscrivent dans l'oniromancie de l'époque - que de la causalité supra-mondaine - ce sont des signes de la progression spirituelle du religieux ${ }^{3}$. Les visions oniriques ou mentales issues de la pratique méditative font partie du tissu mental de l'homme qui constitue l'ensemble de sa vie psychique : ce qui est perçu du monde n'est que ce que la pensée en conçoit, que ce monde soit celui objectif des choses réellement existantes, qu'il soit le flux psychique du sujet lui-même, ou qu'il soit le produit de purs phantasmes.

Ces idées s'étaient largement répandues depuis l'Antiquité. La puissante famille Fujiwara était en effet alors dominante dans le monde politique ainsi que dans le monde littéraire. Le temple tutélaire de cette famille, le Kōfukuji 興福寺, était le siège de l'école Hossō 法相宗, où l'on professait précisément les doctrines du rien-que-conscience [yuishiki 唯識].

3. Voir Mahāyānasamgraha, T.XXXI, n¹594, p. 138a-139b, Lamotte, p. $92-93$ : « Vous dites : "Ces idées ne sont rien qu'idées (vijñaptimātra) puisqu'il n'y a pas d'objet." Quel exemple en a-t-on ? Le rêve (svapna), etc., peut servir d'exemple. Ainsi, dans le rêve, où il n'y a pas d'objet, mais une connaissance sans plus (vijñānamātra), divers objets - couleurs, sons, odeurs, saveurs et tangibles, maisons, bois, terres et montagnes - se manifestent ; pourtant il n'y a là aucun objet réel. Par cette comparaison, on comprendra comment, partout ailleurs [et non pas seulement dans le rêve], il n'y a rien qu'idée (vijñaptimātratā). [Dans la phrase : "le rêve, etc., peut servir d'exemple"], le mot et caetera doit s'entendre d'autres exemples : la magie, le mirage et l'illusion d'optique. Admettons qu'à l'état de veille (prabodha), comme dans le rêve, tout se ramène à une idée sans plus (vijñaptimātra). Mais [au sortir du rêve], on a conscience de ce que le rêve n'était rien qu'idée. Pourquoi une telle conscience ne surgit-elle pas ici également [à l'état de veille] ? - Elle apparaît chez ceux qui sont réveillés (prabuddha) par le savoir de la réalité (tattvajñanna). De même que la conscience [de la non-objectivité des visions du rêve] manque pendant le rêve, mais apparaît après le réveil, de même la conscience [de la non-objectivité des visions du rêve] manque chez ceux qui n'ont pas été réveillés par le savoir de la réalité, mais apparaît chez ceux que ce savoir a réveillés. » 又此諸識皆唯有識都無義故。此中以何爲喻顯示。應知夢等爲喻顯示。謂如夢中都無其 義。獨唯有識。雖種種色聲香味觸舍林地山似義影現。而於此中都無有義。由此喻顯。應 隨了知一切時處皆唯有識。由此等言。應知復有幻註鹿愛舸眩等喻若於覺時一切時處皆如 夢等唯有識者。如從夢覺便覺夢中皆唯有識。覺時何故不如是轉。眞智覺時亦如是轉。如 在夢中此覺不轉。從夢覺時此覺乃轉。如是未得畺智覺時此覺不轉。得真智覺此覺乃轉. 
Prenons un exemple qui illustre notre propos, un poème de Ōshikōchi no Mitsune 凡河内躬恒 (859 ?-925 ?) du Recueil de poésies de jadis et de naguère (905):

«Dans ma somnolence de ne penser

Qu'à toi

Tandis que je rêve endormi,

Ne m'aperçois-je pas que ce que j'ai vu

$\left[\mathrm{Ne}\right.$ vient que] de ma pensée ? $»^{4}$

kimi wo nomi

omohine ni neshi

yume nareba

waga kokoro kara

mitsuru narikeri

君をのみ

思ひ寝に寝し

夢なれば

我が心から

見つるなりけり

Le sens du poème est que les rêves font partie du tissu mental au même titre que les pensées éveillées : si je t'ai vu en rêve c'est parce que je ne pensais qu'à toi ; comme il s'agit de rêves faits durant la somnolence du sommeil, ils ne sont que le produit de mes pensées. Les rêves sont dit-on ce que les hommes voient au moment où ils songent à quelque chose.

Le poème de Mitsune renvoie à un poème d'amour sans titre de Ono no Komachi 小野小町 (IX ${ }^{\mathrm{e}}$ siècle) :

«Comme je dormais

En songeant [à elle],

Serait-ce donc qu'elle m'est apparue en rêve ? [certainement oui]

$\mathrm{Ne}$ me suis-je pas réveillé

Sans savoir que c'était un songe ? $»^{5}$

omohitsutsu

nurebaya hito no

mietsuran

yume to shiriseba

samezaramashi wo

思ひつつ

ぬればや人の

みえつらん

夢としりせば

さめざらましを

4. Kokin wakash $\bar{u}$ 古今和歌集, $\mathrm{n}^{\circ} 608$.

5. Kokin wakashū, n ${ }^{\circ} 552$. Sur ces deux poèmes, voir l'étude de Ishii Kōsei, 2002. 
On peut supposer que les doctrines du rien-que-conscience circulaient comme des leitmotive dans les milieux de cour, à partir de la prédication de moines, non seulement du Kōfukuji, mais de l'ancienne capitale Nara dans son ensemble.

3. Le rêve est pris pour une métaphore du monde empirique de l'illusion. Il est en particulier l'image même de l'expérience du caractère éphémère des choses mondaines, qui se résolvent en un seul instant de pensée. Mais chez des théoriciens comme Myōe et Dōgen, cette métaphore revêt un caractère métaphysique : l'illusoire et le fallacieux du monde ne sont que l'envers de l'authentique, du vrai, ou de la réalité absolue. Seul les distingue le regard porté sur ces deux aspects indissociables et simultanés du réel.

Chez Dōgen, l'illusoire est comparé à un songe, de même que le réel l'est à un état vigile ou éveillé. L'un et l'autre ne sont en dernier lieu que rêve, ce qui ne l'empêche pas de considérer que les expériences passées sont bien réelles à son esprit par le souvenir :

«Pitoyable est le cycle de la naissance et de la mort, il se produit sans trêve Cours de l'illusion comme chemin de l'Éveil avancent [également] dans le rêve

Cependant, il est une chose que je n'arrive point à oublier :

Le bruissement de la pluie nocturne de ma retraite de Fukakusa. »

shōji awaremu beshi kyūshite mata okoru

meito kakuro muchū ni yuku

shikari to iedomo nao wasuregataki koto ari

fukakusa no kankyo yau no koe

生死可憐休又起、

迷途覚路夢中行、

雖然向有難忘事、

深草閑居夜雨声。

4. Le rêve portant sur un signe faste ou un personnage du panthéon bouddhique, est un moyen de pallier l'absence d'un maître, lors d'une ordination religieuse, notamment en période de « Déclin de la Loi »(Mofa, Mappō 末法), selon le Sūtra du Filet de Brahmā (Fanwangjing, Bonmōkyō 梵網經), un célèbre apocryphe chinois qui a connu le plus grand succès au Japon.

6. Variante de Manzan : 唯留一事醒猶記 : «Il n’est qu'une seule chose que je retienne, dont, éveillé, je me souviens encore : » Menzan note que cette mention montre que le poème a été composé à Echizen. Néanmoins l'expression une " affaire », voire celle d'« affaire », ayant le sens de « diffuser la Loi et sauver les êtres » 弘法救生 dans Bendōwa (par exemple, Ōkubo, DZZ, I, p. 741), composé en 1231 (Kangi 3), à l'époque où Dōgen se trouvait à Fukakusa, des interprètes dissocient la signification des deux derniers vers. La lecture de la version du siège (Eiheiji) fait pencher l'interprétation du poème dans le sens d'un souvenir de Fukakusa.

7. EK, X, stance $n^{\circ} 69, \mathrm{DZZ}, \mathrm{IV}, \mathrm{SS}, \mathrm{p} .277$. 
En raison de ce Déclin de la Loi, on en était arrivé à professer un droit égal pour les clercs et les laïcs de se voir administrer des règles disciplinaires communes, celles dites de bodhisattva (bosatsukai 菩薩戒). De fait la coutume s'était largement répandue de conférer l'ordination, non seulement aux religieux, mais aussi aux laïcs dans les milieux de la noblesse, puis, parmi toute la population. On peut donc penser que ce texte est l'une des sources qui donne leur fondement à de nombreuses visions consignées dans les journaux des membres de la noblesse ${ }^{8}$.

5. En accord avec certaines idées de l'ésotérisme, les désirs passionnels, loin de devoir être réprimés, peuvent être débridés afin de se développer en Éveil, grâce à un processus de purification et de sublimation. Un moyen prévalent en est celui de la " conversion des rêves » (yume-tagae 夢違え), qui est parallèle à celui de la 《conversion de direction 》 (kata-tagae 万違え), dans un monde habité de divinités et de forces invisibles, mais agissant au sein de la causalité universelle?

6. Le rêve est aussi un moyen de contourner une réalité trop rugueuse afin de s'entretenir de sujets tabous. En ces périodes du Moyen Âge agitées par les conflits militaires, le récit onirique permettait de mettre en scène des thèmes et des personnages qui, sous couvert de fiction, offraient des alternatives viables en vue d'un monde meilleur. La relation onirique est dans cette optique, non pas authentique, mais largement fictive : sa valeur et son rôle tiennent seulement au degré de croyance que la population accordait aux rêves et au fait que les membres de la noblesse la plus en vue pouvaient en craindre ou en souhaiter les signes auguraux. Ces rêves

8. «Fils de Buddha. Lorsque, après la disparition du Buddha, vous voulez d'une bonne pensée recevoir les règles de bodhisattva, vous prononcez vous-même vos vœux devant une représentation figurée d'un Buddha ou d'un bodhisattva, et recevez les règles. Vous devez vous repentir durant sept jours devant le Buddha afin d'obtenir une vision faste, et de réaliser de ce fait les règles. Lorsque vous n'obtenez pas de vision faste, vous en aurez une sans faute au bout de deux ou de trois semaines, ou au bout d'un an au plus tard. Lorsque vous l'aurez obtenue, vous pourrez alors recevoir les règles devant une représentation figurée d'un Buddha ou d'un bodhisattva. Si vous n'obtenez pas de vision, recevriez-vous les règles devant une représentation de Buddha que vous n'incorporeriez pas les règles. Si [cette vision] s'actualise, vous recevrez d'abord les règles de bodhisattva. Lorsque vous recevez les règles devant un maître, il n'est pas acquis d'avoir une vision. Pour quelle raison? Comme les maîtres précédents se les ont successivement conférées, une vision n'est pas nécessaire. En effet, dès qu'on a reçu les règles devant un maitre, on les a incorporées. C'est en raison d'une pensée de respect qu'on les obtient. Si dans le périmètre de mille lieues $(l i)$, vous ne trouvez pas de maître habilité à conférer les règles, vous pourrez les recevoir en prononçant vous-mêmes les vœux devant une représentation figurant le Buddha et les bodhisattva, à la condition d'avoir une vision.

9. Voir B. Frank, Kata-imi et kata-tagae, Étude sur les interdits de direction à l'époque Heian, Bibliothèque de 1'Institut des Hautes Études Japonaises, Collège de France-Institut des Hautes Études Japonaises, 1998 [1958 $1^{\text {erre }}$ éd.], 288 p. 
intéressent moins la vie psychique intérieure que l'histoire sociale ainsi que les systèmes de représentations collectives de l'époque.

Dōgen qui, de par son obédience bouddhique et qui plus est Zen, n'aurait jamais dû avoir de rêve, a cependant été remarqué par son maître chinois, Rujing 如淨 (1162-1228), pour s'adonner à la méditation assise avec un zèle inaccoutumé, et pour devoir être de ce fait enclin à en obtenir de nombreux signes fastes dans les visions :

«Le maître supérieur m'a prodigué cet enseignement compatissant : “À voir la manière dont tu te comportes dans le pavillon monastique, il me semble que tu t'adonnes à la méditation assise jour et nuit, sans fermer l'œil. Que voilà une excellente chose ! Sans aucun doute, par la suite, il t'arrivera de respirer de fragrantes exhalaisons sublimes sans pareilles en ce monde, ce qui est un signe faste ! Ou encore, tu verras des gouttes d'huile tomber à terre devant tes yeux, ce qui est un autre signe faste. Ou bien encore, tu éprouveras d'autres sensations tactiles, qui seront autant de signes fastes. En de tels moments, adonne-toi à la Voie au moyen de la méditation assise comme pour balayer à toute force le feu qui consume ton chef !" "

La remarque de Rujing ne peut-elle être interprétée dans deux directions différentes ? D'une part, il enjoint certes à Dōgen de redoubler de zèle dans son travail de la Voie par la méditation assise. Mais d'autre part, ne le met-il pas en garde contre un attachement qui pourrait être néfaste envers les visions fastes ? Celles-ci sont en effet des préludes considérés comme nécessaires dans les rituels de confession et de repentance, dans les écoles dites doctrinales $k y \bar{o} k e$ 教家, que Rujing et Dōgen connaissent également bien ${ }^{10}$. Or, le mot d'ordre de Rujing repris par Dōgen de ne pas brûler de l'encens, ni de révérer des images pieuses, mais de «s'asseoir directement en concentration mentale sans plus », qui est une critique de ces pratiques préparatoires, ne va-t-elle pas de pair avec une dévalorisation des visions, fussent-elles fastes, au cours de ces préambules jugés aussi interminables qu'inutiles, voire que nuisibles ? ${ }^{11}$

10. À propos de ces rituels chez Zhiyi 智顗 (538-597) le grand théoricien du Tiantai, voir Kuo Liyin, Confession et contrition dans le bouddhisme chinois du $v^{e}$ au $X^{e}$ siècle, Publication de l'École Française d'Extrême-Orient, Monographie n 170, Paris, 1994, p. 102-104.

11. Le Hōkyōki éclaire bien ici la logique de pensée de Rujing et de Dōgen. Pour ceux qui mettent en doute l'authenticité des dires du Hōkyōki et en font un recueil de propos tardif de Dōgen, il va de soi que ce passage pourrait n'être qu'une manière de justifier a posteriori le récit onirique que Dōgen aurait caché une quinzaine d'années durant, récit qui ne serait que fictif. Nous ne partageons pas cette opinion et croyons que, à l'exception de l'Introduction, le Hōkyōki est un recueil de sa jeunesse qui, au contraire, illustre de manière remarquable l'attitude de Dōgen à l'égard des rêves dont il se méfie, mais qu'il ne peut s'empêcher d'avoir, dans la meilleure tradition sinojaponaise, sinon japonaise tout court. 


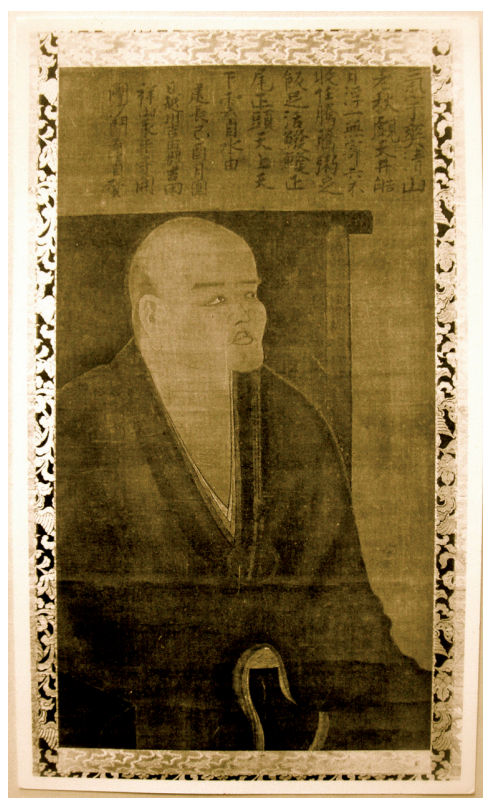

FIG. 1. - Portrait de Dōgen contemplant la lune (1249, Hōkyōji, copie de Giun, fin XIII ${ }^{\mathrm{e}}$-début XIV ${ }^{\mathrm{e}}$ siècle).

C'est précisément au cours de son séjour en Chine, qui s'est déroulé entre 1223 et 1227 , que Dōgen a eu le seul rêve dont l'authenticité n'est pas sujette à caution. Il est relaté seize années après coup dans le sermon «Livre généalogique » (Shisho 嗣書) (1241), qui constitue à lui-même plutôt une monographie écrite, destinée à circuler de façon interne au sein de sa communauté, qu'un discours de prédication publique. C'est en effet l'un des très rares autographes de ce moine qui nous soit parvenus en son intégrité. Au début de l'année 1225 (la première année de l'ère chinoise Baojing), Dōgen s'en retourne au Tiantongshan, où Rujing avait été accueilli comme supérieur à la fin de l'année précédente. En cours de route, il rallie le Wanniansi 萬年寺, où il rencontre le supérieur, Yuanzi 元穿 (?-?), qui lui montre, fait remarquable entre tous car sans précédent, le Livre généalogique de sa tradition. À la suite de quoi, Dōgen fait un détour par le Hushengsi 護聖寺 du mont Damei 大梅山 (littéralement, le Prunier géant) avant d'aller rencontrer pour la première fois Rujing, le $1^{\mathrm{er}} \mathrm{du}$ cinquième mois au Tiantongshan, auprès duquel il réalisera l'Éveil et obtiendra la reconnaissance autorisée de transmettre ses enseignements. C'est donc un songe 


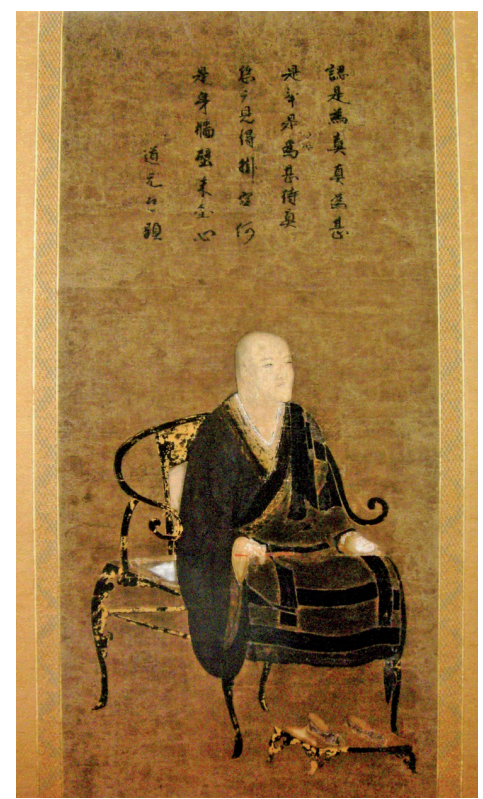

FIG. 2. - Portrait de Dōgen (Eiheiji, XVI ${ }^{e}$ siècle ?).

\section{prémonitoire de cette rencontre, qui marque un tournant décisif dans} sa vie religieuse. Il le relate ainsi :

« Par la suite, durant l'ère Baojing [1225-1227], tandis que je pérégrinais aux monts Tiantai et Yantangshan, j'ai atteint le monastère Wanniansi à Pingdian. Le supérieur en était à cette époque le maître Yuanzi de Fuzhou. Il assumait cette fonction à la suite de la retraite du doyen Zongjian, et avait redonné le plus grand succès aux assemblées monastiques de prédication. Tandis qu'on en était aux présentations, on échangeait des propos sur les manières de faire des Buddha-patriarches depuis les temps jadis et, lorsqu'on en arriva à traiter de l'anecdote de la succession chez Dawei et Yanshan, le doyen [Yuanzi] s'enquit: "Avez-vous déjà vu le Livre généalogique que j'ai chez moi ?" Je lui répondis : "Comment donc cela serait-il possible ?" Sur ce, le doyen se leva sur le champ et déclara en brandissant le Livre : "Je ne l'ai jamais montré à qui que ce soit, fût-ce à un ami intime ni même à un moine desservant depuis de longues années ! Il est l'enseignement des Buddha-patriarches". Or, ces jours-ci je suis allé à une ville où je suis resté afin de rendre visite au préfet. J'ai alors eu le rêve suivant: "Un moine qui me semblait être le maître de Dhyāna Fazhang (752-839) du mont Damei [Grand Prunier], me tendit une fleur de prunier tout en me déclarant : 'S'il se trouve un homme authentique qui aura traversé les océans en bateau, donne-la lui sans lésiner !' Et il me donna la fleur." Yuanzi ne put réprimer, malgré lui, l'envie de clamer ce poème : 
"Celui qui n'a pas enfourché le pont d'un navire,

Qu'on se le dise : Je lui assénerai trente coups de bâton!"

Mais en moins de cinq jours, il m'a été donné [à moi Yuanzi] d'avoir une entrevue avec vous [Dōgen]. Non seulement vous [Dōgen] avez enfourché le pont d'un navire, mais encore ce Livre généalogique est tissé avec le motif du prunier. Vous êtes donc bien celui dont Damei m'avait parlé dans mon songe. C'est parce qu'il y a coïncidence exacte avec mon rêve que je vous ai montré mon Livre.

Je [Dōgen] n'arrivais pas à y croire ! J'aurais dû l'implorer de me donner le Livre généalogique mais je ne fis que le saluer en brûlant de l'encens et le révérer en faisant une offrande [de monnaie]. À ce moment-là, le préposé à l'encens, du nom de Fa.ning, confia avoir vu à ce moment-là aussi pour la première fois le Livre généalogique.

Je [Dōgen] me dis à part moi-même que jamais je n'aurais eu connaissance d'un tel Livre généalogique sans l'aide gracieuse des Buddha-patriarches. Par quel bonheur un simple d'esprit tel que moi, venant de la contrée marginale du Japon, ai-je pu le voir à discrétion! J'en essuyai les larmes qui imprégnaient mes manches. À ce moment-là, la cellule de Vimalakīrti $[=\mathrm{du}$ supérieur $]$ ainsi que le grand pavillon monastique ${ }^{12}$ se vidèrent de monde et le plus grand silence se fit.

Le Livre généalogique était écrit sur un fond blanc tissé de motifs de fleurs de prunier. Il faisait plus de neuf pouces de long sur plus d'une toise de large. Son axe était de gemmes jaunes et sa couverture de brocard.

Sur la route me menant du mont Tiantai au mont Tiantong, j'avais fait une halte au monastère du Hushengsi sur le mont Damei [Le Grand Prunier], Cette nuit-là, j'avais vu dans un rêve surnaturel le maître-patriarche Damei [Grand Prunier] venir me donner une fleur de prunier épanouie. C'est la chose la plus digne de confiance que de bénéficier de la bienveillance d'un patriarche [comme Damei] ! La branche de prunier faisait un pied de long et de large. La fleur méritait bien le qualificatif de fleur d'Udumbara. Il s'agissait assurément d'une même et seule réalité aussi bien en rêve que dans la veille. Jamais je n'en ai parlé aux autres, tant quand j'étais en Chine qu'après être revenu au Japon. »

Il semble que Dōgen avait le sentiment d'une mission à accomplir : celle de transmettre, toutes affaires cessantes, la Loi au Japon. C'est ainsi que se laisse interpréter sa quête, si l'on en suit les intentionnalités. Le voyage qu'il a entrepris de faire en Chine n'avait pas d'autre visée. À la différence d'autres religieux qui rapportaient livres et reliques, Dōgen s'en retourne sans autre bagage que l'enseignement vivant dont il a pris une connaissance intime de son maître chinois qui l'illustrait : rien moins qu'une entrevue de visu (menju 
面授), et non pas une simple connaissance livresque ni le témoignage matériel d'un pèlerinage, n'est à ses yeux nécessaire ${ }^{13}$.

L'ardeur de sa recherche est mise en évidence par le caractère dramatique de son voyage : il s'inscrit dans la continuité des volontés du troisième shōgun, Minamoto no Sanetomo 源實朝 (1192-1219) qui avait entrepris une telle traversée en Chine sur les trace du grand moine Yōsai (/Eisai) 榮西 (1141-1215), l'introducteur du Zen Rinzai au Japon, volontés violemment contrecarrées par son assassinat en 1219. Dōgen prend le départ en 1223 avec son maître Myōzen 明全 (1184-1225), du temple Kenninji, alors que le propre maître de celui-ci, Myōyū 明融 (?-1223), est agonisant : leur ardeur est si pressante que les deux moines n'attendent pas la mort de Myōyū pour quitter leur pays. Dōgen voit Myōzen trépasser sous ses yeux en Chine, en 1225, organise sur place ses funérailles à son intention et revient au Japon avec ses reliques ; il est notable que, contrairement à ses prédécesseurs qui revenaient de Chine avec des restes matériels du Buddha, ce sont les seules reliques qu'il rapportera de Chine, signe qu'un maître humain a une valeur égale, sinon supérieure à ses yeux à celle d'un Buddha impersonnel, lointain ou pur idéal abstrait. Il quitte le Continent tandis que son maître chinois est à son tour malade, assez proche de sa fin. Enfin, à son arrivée au Japon, ne s'assigne-t-il pas comme devoir de ne pas assister aux funérailles de son propre père présumé, Minamoto no Michitomo 源通具, survenue le 2 du neuvième mois de 1227, par respect de la stricte interdiction de Rujing de s'approcher des grands de ce monde (Michitomo était grand chancelier, dainagon) ? Son œuvre missionnaire prime toute considération concernant la vie humaine, la sienne propre comme celle de ses maitres ou de ses proches.

Dōgen voulait se rendre en Chine afin d'obtenir un enseignement effectif de la Loi, celui d'un maître vivant, et en être reconnu avant de revenir au Japon pour le transmettre. Les Notes de l'ère Baojing (le Hōkyōki 寶慶記), rédigées par Dōgen au cours de son voyage en Chine, relatent cette aventure humaine et religieuse dans ses ressorts internes. Des passages du Thesaurus de l'Eil de la vraie Loi y font de même référence, d'une façon circonstanciée. Tel est le cas du rêve de Dōgen qui prend place à une jonction cruciale de sa vie : il se rend au Tiantongshan où réside Rujing, son futur maître qui le reconnaîtra 2005 .

13. Voir Fr. Girard, «Quête et transmission de reliques de la Chine au Japon au XIII ${ }^{\mathrm{e}}$ siècle », 


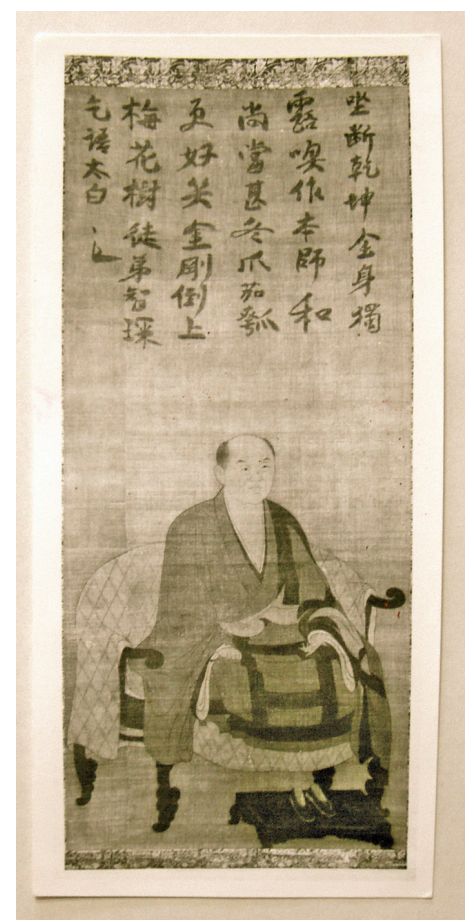

FIG. 3. - Portrait de Rujing (Hōkyōji).

comme dépositaire de son enseignement, et il prend connaissance d'un Livre généalogique de l'écoles du Dhyāna, texte capital qui lui en ouvre par préfiguration l'accès. Le rêve est le signe que la quête est sur le point d'aboutir. Il appartient à la catégorie des « rêves réels » ou « de réalisation » (genmu 現夢), qui se caractérisent par la concrétisation des volontés du rêveur, et qui influent sur le cours des événements intervenant dans la vie réelle. Il définit un événement mental qui infléchit sur le cours des choses de manière décisive, tout autant que le ferait un acte de décision conscient.

Le sermon "Livre généalogique » a été rédigé par Dōgen le 7 du troisième mois de 1241 (Ninji 2), à une époque où il venait de fonder le monastère Kōshōji de Fukakusa aux abords de Kyōto. Il est l'un des rares sermons du Thesaurus à n'avoir pas été l'objet d'une prédication publique destinée à l'assemblée monastique ou à des laïcs. C'est donc un document privé, à usage interne, comme le sont d'autres textes parmi les plus précieux pour une communauté religieuse qui restent sans être divulgués. Le Journal des rêves de 
Myōe en est un exemple comparable. Ce caractère en fait l'un des textes les plus importants de l'œuvre de Dōgen, de ceux qui sont l'objet d'une transmission privée, voire secrète.

Sur le plan du contenu, le "Livre généalogique » s'inscrit dans la continuité immédiate d'un sermon rédigé peu auparavant, «Buddha-patriarches »(Busso 佛祖), le 3 du premier mois de la même année. Il s'agit là aussi pour Dōgen de montrer qu'il hérite en droite ligne de la Loi du Buddha historique et, par delà, des Sept Buddha du passé, jusqu'à lui-même en passant par Rujing. Dans les conceptions de Dōgen, Buddha et patriarches sont mis sur un même plan et donc sur un strict pied d'égalité : selon un processus de désacralisation intégrale, les Buddha ne sont pour lui autres que des hommes qui ont eu l'expérience de l'Éveil au même titre que les patriarches, si bien qu'ils ne désignent qu'une seule et même catégorie d'êtres humains. Dōgen, qui a comme l'on dit réalisé « la grande affaire de la Loi bouddhique " grâce à Rujing, est donc en continuité avec les Buddha-patriarches qui forment une lignée ininterrompue dont lui-même est actuellement l'ultime chaînon. En héritant du Livre généalogique en Chine, qui prouve cette continuité, il devient de manière incontestable dépositaire de la vraie Loi qu'il a introduite au Japon. Ainsi qu'il se qualifie lui-même, il est à proprement parler « le moine Dōgen qui est entré dans la Chine des Song afin de transmettre la Loi » (Nissō denpō shamon Dōgen 入宋 傳法沙門道元). Tel est le sens de l'existence du 《 moine » Dōgen. S'il n'a ni à le clamer ni à le proclamer à tout venant, il importait néanmoins de consigner cet événement de première importance par écrit dans un ouvrage à ne divulguer que parmi la communauté qui est soudée par cette connaissance intime. C'est précisément dans ce sermon à caractère secret que Dōgen relate son songe, sans en avoir jamais parlé à quiconque : il suit une tradition japonaise de silence à l'égard des rêves les plus chers qui révèlent les volontés profondes de leurs auteurs.

Par la suite Dōgen rédige un sermon intitulé « Fleur de prunier » (1243), dans lequel il reprend la thématique de l'éclosion de l'Éveil et la transmission de la Loi, à l'aide de la métaphore de la fleur. Il compose également des stances poétiques sur la « fleur de prunier». Par ailleurs, il approfondit sa réflexion sur la nature du rêve dans le sermon « Prêcher le rêve dans le rêve ", qui vient asseoir en arrièrefond le bien-fondé de sa vision faite en Chine : ne l'a-t-il pas rédigé trois mois après avoir reçu en mains propres un exemplaire des Logia de Rujing arrivés de Chine, qui lui ont donné l'occasion se 
remémorer d'une seule bouffée son expérience chinoise ? Il établit dans ce sermon une continuité entre rêve et veille, dans la lignée des conceptions japonaises de son époque, à l'aide d'une dialectique du Zen héritée de l'image du « rêve du papillon » du Zhuangzi :

« Rêve et veille sont dès le départ de nature identique, ils sont la réalité même. La Loi bouddhique, fût-elle métaphorique, est par définition la réalité même. Elle n'est déjà plus une métaphore et c'est ce qui se passe en rêve qui est la réalité authentique de la Loi bouddhique. $\gg^{14}$

Dōgen situe le rêve comme en continuité avec l'état vigile : même en songe il est possible selon lui de concevoir la pensée de l'Éveil. Dans le sermon intitulé « Conception de la pensée d'Éveil suprême » (Hotsu mujōshin 発無上心)(1244), l'état de rêve est un état subnormal et subconscient, puisqu'il est mentionné en continuité avec celui de l'ivresse :

« Dans toutes les conditions et circonstances où les choses se combinent, il y a toujours conception de la pensée de l'Éveil, que ce soit en rêve ou en état d'ébriété, il y a conception de la pensée d'Éveil et réalisation de l’Éveil. $»^{15}$

Cette idée implique que la pensée de l'Éveil et l'Éveil peuvent se produire en n'importe quel état, aussi défavorable en apparence soit-il, et qu'il y a continuité entre l'état vigile et l'état de somnolence onirique, qui ne sont que deux facettes, extrêmes, d'un même continuum psychique. Sur ce fondement épistémologique, la réalité donnée à l'expérience, qui est une et identique, devient la quête de la vérité ultime des choses. C'est l'effort déployé afin de percer à jour la vérité sous le voile des apparences, qui prime.

Dōgen développe cette dernière idée dans ce sermon, «Prêcher le rêve dans le rêve » (muchū setsumu 夢中説夢), du 21 du onzième mois de 1242, exposé avec les Logia de Rujing entre ses mains ${ }^{16}$. Il ne fait aucun doute que cet événement a été pour lui une expérience comparable à celle de la madeleine proustienne, qui lui a permis de se remémorer ses entretiens avec Rujing. Le style de ce sermon s'en ressent fortement, qui d'ailleurs cite un passage des Logia. Dōgen n'hésite pas à expliquer que la Loi peut sans inconvénient être prêchée en état de rêve. Cette prédication ne cherche pas à dégager un principe ou une loi au sein des phénomènes qui

\footnotetext{
14. DZZ, I, p. 244.

15. DZZ, I, p. 526.

16. Montée en salle du 6 du huitième mois de 1242 .
} 
sont aussi fugaces qu'un songe. Au contraire, en surenchérissant en somme sur l'irréalité de l'irréel, elle laisse entrevoir les choses telles qu'elles apparaissent. Le rêve est une image courante, on l'a vu, de l'idée que les choses sont au fond sans substance et sont dépourvues d'être en soi. Ce que Dōgen entend dire en premier dans ce sermon, c'est que, parmi le monde phénoménal qui est tel un songe, il serait vain de rechercher la moindre consistance, s'agissant même des choses de la Loi : leur apparition est le dernier mot de leur être, tout provisoire soit-il. La réitération de l'inconsistance des choses équivaut à porter leur évanescence à l'infini.

Mais cette vision radicalement purgative des choses les manifeste en retour dans le plein éclat de leur réalité qui est celle de l'instantané, sans plus, de leur émergence. C'est de cette façon que tout un chacun voit les choses telles qu'elles sont. Dōgen, fidèle à ses principes, identifie l'explicitation du rêve dans le rêve à la réalisation réaffirmée au sein de la réalisation :

«Qui donc douterait que le rêve est l'Éveil ? Comme il n'est pas la moindre place au doute, qui donc l'admettrait ? [Personne car personne] n'est mû par une telle reconnaissance ${ }^{17}$. Étant donné que cet Éveil suprême est l'Éveil suprême, le rêve est qualifié de rêve. Il est un rêve "dans" [la prédication du rêve dans le rêve] ${ }^{18}$, il est une prédication du "rêve", il est un rêve qui est "prédication" et il est un dedans du "rêve" [qu'est l'Éveil suprême]. Sans un "dedans du rêve" 19 , il n'est pas de prédication du rêve ${ }^{20}$, sans prédication du rêve $e^{21}$, il n'est pas de "dedans du rêve" ${ }^{22}$, sans "prédication du rêve" ${ }^{23}$, il n'est nul Buddha, sans un dedans du rêve ${ }^{24}$, aucun Buddha n'apparaîtra dans le monde ni aucune motion de la roue de la sublime Loi ne se produira. »

\section{Conclusion}

Le récit onirique de Dōgen relatif à la fleur de prunier se présente comme un signe faste annonçant sa rencontre décisive avec Rujing qui, elle, a une véritable consistance humaine, doctrinale et religieuse. Le rêve est la prémonition d'un événement réel : il ne diffère

17. L'être humain n'est pas mû par un acquiescement spéculatif ni cognitif, mais par une expérience.

18. Rêve qui est l'Éveil suprême.

19. Un dedans de l'Éveil suprême.

20. Prédication de l'Éveil suprême.

21. Prédication de l'Éveil suprême.

22. Dedans de l'Éveil suprême.

23. Prédication de l'Éveil suprême dans l'Éveil suprême.

24. Dedans de l'Éveil suprême. 
pas de la réalité, ainsi que le note Dōgen lui-même. Mais en l'occurrence, ce qui donne une consistance accrue à l'événement est l'aspect fortuit de la coïncidence entre le rêve de Dōgen et celui du supérieur du Wanniansi, qui rapportent strictement la même chose et viennent exactement se superposer. Cet événement « inouï », « inimaginable », « jamais vu auparavant » (misou), comme il se plaît à le souligner, annonce un événement non moins " inouï », celui d'avoir pu rencontrer Rujing, au terme de son voyage. Une fois que l'événement s'est produit, en deux phases - un signe annonciateur, une rencontre réelle qui porte ses fruits -, Dōgen a accompli sa tâche et sa mission en Chine : il ne lui reste plus qu'à s'en retourner au Japon, ce qu'il fait.

Le voyage de Dōgen en Chine définit, avant tout, un itinéraire mental. À la différence de ses prédécesseurs japonais qui tenaient des chroniques détaillées, datées et circonstanciées au jour le jour de leur séjour continental, Dōgen a relaté ses seuls dialogues avec Rujing, sans presque jamais décrire les circonstances de leur rencontre ni mentionner de date. Il inaugure, dans l'histoire japonaise, un type de voyage sur le Continent, nouveau par son aspect radical. Sa quête de la Loi se traduit, non pas à travers des textes canoniques, des reliques rapportés au Japon à l'issue d'une transaction commerciale, ou encore un parcours de pèlerinage - tel a été le cas de Yōsai, qui a servi de modèle pour la cour shôgounale -, mais par la volonté de toucher du doigt un enseignement vivant grâce à la rencontre d'une personne qui en est le dépositaire. Jeune étudiant, un peu tête brûlée, il se rend en Chine résoudre ses doutes concernant des pratiques qu'il juge laxistes et dont il est le témoin au Japon : convaincu que les règles disciplinaires doivent être respectées dans leur esprit, il croit en trouver une illustration dans un Zen rigoriste et abrupt.

Les lignées généalogiques, grâce auxquelles un enseignement humain et vivant se transmet en substance de personne à personne, est une constante des conceptions japonaises. Les transmissions secrètes, ésotériques ou orales, en sont un mode d'expression. Le rêve de la fleur de prunier qui « est une réalité », consigné quelque seize années après qu'il l'a eu expérimenté, entre dans la lignée des récits oniriques qui influent sur le cours des choses ; il rejoint également une autre thématique de Dōgen : les images, les métaphores et les expressions symboliques sont toutes des choses réelles. Chez lui la représentation vaut la substance des choses. Ce récit éclaire sans nul doute les conceptions de ce religieux, inconnu en son temps et ignoré de l'histoire - les sources qui lui sont contemporaines sont 
muettes à son égard -, et devenu de nos jours peut-être le plus emblématique des religieux du Japon. Nul besoin de souligner également que les thèmes poétiques développés autour du prunier et de la fleur de prunier chez Dōgen - ils sont nombreux - puisent à cette anecdote comme à sa source et en développent le halo sémantique à loisir. 


\section{BIBLIOGRAPHIE CHOISIE}

Carrique, Pierre, Rêve, vérité, Essai sur la philosophie du sommeil et de la veille, NRF Essais, Gallimard, Paris, 2002, 395 p.

Dictionnaire des Mythologies, sous la direction de Yves Bonnefoy, Paris, Flammarion, 1981.

D'Hervey de Saint-Denys, Les rêves et les moyens de les diriger. Observations pratiques, Édition intégrale, Oniros, Île saint-Denis,

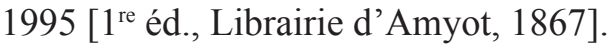

Frank, Bernard, Kata-imi et kata-tagae, Etude sur les interdits de direction à l'époque Heian, Bibliothèque de l'Institut des Hautes Études Japonaises, Collège de France-Institut des Hautes Études

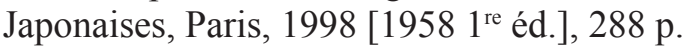

Girard, Frédéric, Un moine de la secte Kegon à l'époque de Kamakura, Myōe (1173-1232) et le Journal de ses rêves, Paris, Publications de l'École française d'Extrême-Orient, volume CLX, Paris, 1990, LXXXVIII+598 p.

, "Le chapitre Vie et mort du Thésaurus de l'œil de la vraie Loi de Dōgen », Cahiers d'Extrême-Asie n 9, Mémorial Anna Seidel, Tome II, Kyōto, 1996-1997, p. 299-311. , « Le samādhi de réflexion sigillaire océanique chez Dōgen (1200-1253) », dans Le Vase de béryl, Mélanges offerts à Bernard Frank, Paris, Éditions Picquier, 1997, p. 75-86.

, « Myōe shōnin muki » [« Le Journal des rêves de Myōe »], dans Ibunka tono deai [Rencontre avec une culture étrangère], Encounters between Cultures, Papers from the Ferris University International Conference on Japanese Literature, Yokohama, 2003, p. 100-118;205-206; 227-228.

, "Quête et transmission des reliques de la Chine au Japon au $\mathrm{XIII}^{\mathrm{e}}$ siècle », dans Les objets de la mémoire. Pour une approche comparatiste des reliques et de leur culte, $\mathrm{Ph}$. Borgeaud et Y. Volokhine (éd.), Studia Religiosa Helvetica 10, Peter Lang, Bern-Berlin, 2005, p. 149-179.

Heine, Stevens, A Dream Within a Dream, Studies in Japanese Thought, Peter Lang, New York, 1991.

, « The Dōgen Canon : Dōgen's Pre-Shōbōgenzō Writings and the Question of Change in His Later Works », Japanese Journal of religious Studies 24/1-2, Spring, 1997. 
, Did Dōgen go to China? What he Wrote and When he Wrote it, Oxford University Press, Oxford-New York, 2006.

Kawai, Hayao, The Buddhist Priest Myōe : A Life of Dreams, Translated and Edited by Mark Unno, The Lapis Press, Venice, 1992.

Kawatō Masashi 河東者, Nihon no yume shinkō - shūkyōgaku kara mita Nihon seishinshi - 日本の夢信仰 - 宗教学から見た日本 精神史 - [Les croyances oniriques au Japon - Histoire mentale du Japon du point de vue des sciences religieuses], Tamagawa daigaku shuppanbu 玉川大学出版部, Tōkyō, 2002.

Kuo Liyin, Confession et contrition dans le bouddhisme chinois $d u v^{e}$ au $X^{e}$ siècle, Publication de l'École Française d'ExtrêmeOrient, Monographie $\mathrm{n}^{\circ}$ 170, Paris, 1994.

Kuramoto Kazuhiro 倉本一宏, Heian kizoku no yume bunseki 平安貴族の夢分析 [Analyse des rêves chez la noblesse de Heian], Yoshikawa kōbunkan 吉川弘文館, Tōkyō, 2008.

Liou Wenying 劉文英, Chūgoku no yume handan 中国の夢制断 [Oniromancie chinoise], traduction par Yuasa Kunihiro 湯浅邦弘, Tōhō shoten 東方書店, Tōkyō, 1997.

Matsuo Kenji 松尾剛次, « Muki no ichisekai - kōsō nikki to jisei jukai - 》夢記の一世界[《Un monde de chronique onirique - les journaux de visions fastes et les auto-ordinations »], dans Sonoda Kōyū 薗田香融, Nihon bukkyō no shiteki tenkai 日本仏教の史 的展開 [Développement historique du bouddhisme japonais], Hanawa shobō 塙書房, Tōkyō, 1999.

Nakimovitch, Pierre, Dōgen et les paradoxes de la bouddhéité, École Pratique des Hautes Études, IV ${ }^{\mathrm{e}}$ section, Paris-Genève, Droz, 1999.

Schmitt, Jean-Claude, Le corps des images, Essais sur la culture visuelle au Moyen Âge, Le temps des images, Paris, Gallimard, 2002.

Sugawara Akihide 菅原昭英, « Yume wo shinjita sekai - Kujō Kanezane to sono shūi - 夢を信じた世界一九条兼實とその 周囲 - » [《Un monde qui croyait aux rêves - autour de Kujō Kanezane »], dans Les couches profondes du monde religieux [Shūkyō sekai no shinsō 宗教世界の深層], Nihongaku 日本學, $\mathrm{n}^{\circ}$ 5, octobre 1984.

Sugawara Akihide 菅原昭英, Idem, « Dōgen zenji no yumegatari - Shōbōgenzō yori » 道元禅師の夢語り - 正法眼藏より - [« Le récit onirique chez le maître de Dhyāna Dōgen - à partir du Thésaurus de l'œil de la vraie Loi-»], Azuma Ryūshin hakase koki kinen ronshu - Zen no shinri to jissen - 東隆畺博士古希 
記念論集 [Recueil commémoratif pour le $70^{e}$ anniversaire du Docteur Azuma Ryūshin - La vérité et la pratique du Dhyāna], Shunjūsha 春秋社, Tōkyō, 2005, p. 47-63.

" Nansō zenrin ni okeru yume-gatari - Mujun shiban no baai 》南宋禅林における夢語り無準師範の場合 [《Récits de rêves dans les temples de Dhyāna des Song du Sud - Le cas de Wuzhun shifan »], Komazawa joshi daigaku kenkyū kiyō 駒沢女子大学研究紀要, $\mathrm{n}^{\circ} 11$ et 12, décembre 2004, p. 1-15, et décembre 2005, p. 1-14.

Tamai Kōsuke 玉井幸助, Nikki bungaku gaisetsu 日記文學概説 [Introduction à la littérature de journaux privés], Kokusho kankōkai 國書刊行會, Tōkyō, réédition 1982 [1945].

Yasuraoka Kōsaku 安良岡康作, Chūseiteki bungei no rinen 中世的 文芸の理念 [Les idéaux des arts littéraires médiévaux], Kasama shoin 笠間書院, Tōkyō, 1981.

\section{ABRÉVIATIONS}

DZZ : Dōgen zenji zenshū 道元禪師全集 [Euvres complètes du maître de Dhyāna Dōgen], par Ōkubo Dōshū 大久保道舟, 2 volumes, Tōkyō, Chikuma shobō, $1^{\text {re }}$ édition 1970, réimpression, Rinsen shoten 臨川書店, Kyōto, 1989.

DZZ 2: Dōgen zenji zenshū 道元禪師全集 [Euvres complètes du maître de Dhyāna Dōgen], par Kagamishima Genryū, Sakai Tokugen et Sakurai Shūyū, 7 volumes, Shunjūsha 春秋社, Tōkyō, 1988-1993.

EK : Eihei kōroku 永平廣録, Notes étendues du Eiheiji, DZZ, II.

Monge : Hōkyōki monge 寶慶記聞解 [ou Hōkyōki zuimonki 寶慶記 隨聞記] [Explication des Dialogues de l'ère Hōkyō]. Commentaire doctrinal oral de Menzan Zuihō 面山瑞方 (1683-1769), recueilli par Fuzan Gentotsu 斧山玄鍴 (金出) (mort en 1789). Édité pour la première fois par Kochi Chijō 古知知常 en 1868, nous avons consulté une version imprimée sous ce titre en 1878, en deux volumes, chez Morie zōhan 森江藏版, Tōkyō. SSZ, IX (Chūge 註解, Commentaires, IV), 1936.

SGCZ : Shōbōgenzō chūkai zensho 正法眼藏註解全書 [Collection complète des commentaires du Thésaurus de l'oil de la vraie Loi], Nihon bussho kankōkai 日本仏書刊行会, 11 volumes, Tōkyō, réimpression 1965-1968.

T. : Taishō shinshī daizōkyō 大正新修大藏經. 


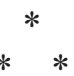

M. Jean-Noël RoBERT intervient après cette note d'information. 